\title{
Wear of sharp aggregates in a rotating drum
}

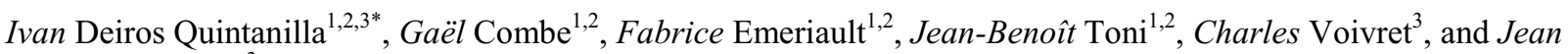 \\ François Ferellec ${ }^{3}$ \\ ${ }^{1}$ Université Grenoble Alpes, 3SR, F-38000 Grenoble, France \\ ${ }^{2}$ CNRS, 3SR, F-38000 Grenoble, France \\ ${ }^{3}$ SNCF Réseau, Direction Ingénierie \& Projets, 93574 La Plaine Saint Denis, France
}

\begin{abstract}
Aggregates constituting ballast layer wear due to the continuous passage of trains and during the necessary maintenance operations of the track. In order to develop efficient solutions for ballasted tracks design and maintenance, a proper knowledge of the degradation laws of ballast grains is needed. In tribology, the amount of wear due to friction when two surfaces are in contact is classically predicted by Archard's equation. However, due to the continuous evolution of grain angularity and roughness, at the macro-scale wear coefficient cannot be assumed to remain constant, but will depend on the state of degradation of the grain surface. In order to adjust the model to this particular case, the Micro-Deval Attrition test is used. The rotating drum is stopped at intermediate stages and the amount of generated fine particles is measured. Thus the curve of mass loss along time is built. These results are then linked to Archard's model using the values of contact forces and relative displacements extracted from discrete element simulations. Finally, a morphology analysis is performed tracking shape and roughness parameters at different stages of degradation using X-ray tomography and a laser profilometer.
\end{abstract}

\section{Introduction}

In ballasted railway tracks, ballast layer plays a crucial role in the transmission of dynamic stresses imposed by the circulation of trains to the geotechnical structure supporting the track. The performance of ballast strongly depends on the shape, size and mineralogical nature of the grains composing it. Throughout the life of the track ballast particles wear, due to the constant friction between grains, steadily reducing the angularity and surface roughness of the grains and producing very fine particles $(\mathrm{d}<0.5 \mathrm{~mm})$ that will eventually induce a loss of track performance. The interlock between particles is reduced limiting the anchorage of sleepers and the transfer of loads to the platform, and the presence in excess of fine particles renders maintenance operations ineffective and reduces the permeability of the track.

After some years of service of the first high-speed service in France, ballast has proven not to be resistant enough, showing a faster degradation than expected. This has been partially compensated over the years by the strict selection of good quality granite for the aggregates. This selection is performed in the quarry, where some mineral samples are subjected to an attrition test called Micro-Deval (MDA) [1, 2]. During this test, $10 \mathrm{~kg}$ of grains are turned at $100 \mathrm{rpm}$ inside a drum during 140 minutes. Particles retained at the $1.6 \mathrm{~mm}$ sieve are then weighted and compared to the initial weight through a dimensionless coefficient $C_{M D A}$ determining the resistance to abrasion of the material.

\section{Archard model}

Wear produced by particles in contact is a very complex process depending on the loading conditions at the contact scale. In tribology, Archard model [3] has been classically used to predict the flux of eroded mass when two surfaces in contact move relatively one to the other. Archard assumes that the volume $W$ of fine particles produced is proportional to the normal force $\left(f_{n}\right)$ and the relative displacement between surfaces $(s)$.

$$
W=(k / H) \cdot f_{n} \cdot s
$$

The product $f_{n} \cdot s$ is directly related to the work produced by friction forces. On the other side, the proportionality is often expressed as $k / H$, where $H$ is the hardness of the softest material and $k$ is a dimensionless constant called wear coefficient. In the case of study, $H$ can be considered as constant since the material is granite for both surfaces and does not change its properties when eroded. However, surfaces in contact suffer changes in shape, losing their roughness and angularity, and consequently reducing the severity of wear at the contact interface. At the macro-scale, wear coefficient cannot be assumed to be constant, but will depend on the state of degradation of the surface.

In order to adjust Archard model to the particular case of irregular sharp aggregates, the MDA test is used, linking the variables extracted from discrete element simulations of the test with the results of the real experimental test. 


\section{Micro-Deval}

\subsection{DEM simulation}

In order to better understand the wear process occurring within the Micro-Deval device, a discrete element simulation of the test [4, 5], using a Non-Smooth Contact Dynamics approach (NSCD) [6], has been performed and compared with the experimental results.

The drum has been numerically built following the European code $[1,2]: 40 \mathrm{~cm}$ long with a $20 \mathrm{~cm}$ inner diameter. The grains have been randomly chosen from a database of 1000 different modelled ballast grains. This database has been built following the correct ballast grain-size distribution using polyhedral shapes containing from 12 to 70 faces and from 8 to 37 vertices. In order to cover the required $10 \mathrm{~kg}$ of ballast, 109 grains are required with a density of $2700 \mathrm{~kg} / \mathrm{m}^{3}$.

Collisions are considered perfectly inelastic and grains are modelled as rigid bodies. Wear is not modelled during the simulation. Thus, only 35 seconds of test have been simulated, since system conditions remain the same along time.

Friction coefficient between ballast grains has been stablished as 0.8 , following the results of experimental tests (Figure 1), and between ballast and the metallic drum arbitrarily set at 0.4 . It is noteworthy that friction coefficient does not change significantly between new and eroded grain surfaces, especially when dealing with small relative displacements.

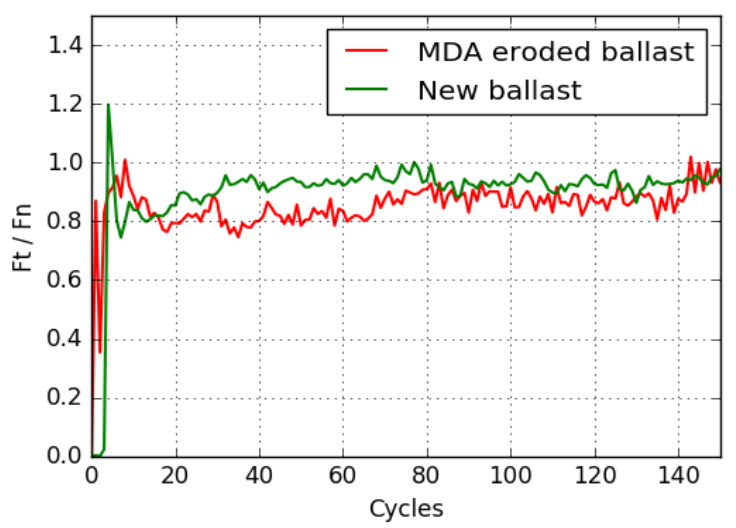

Fig. 1. Evolution of the friction coefficient between two grains along shearing cycles in BCR3D shearing device [7].

Simulations show that the MDA test is a very dynamic process in which grains turn very fast at almost the same speed as the cylinder. The grains kinematics analysis highlights two plausible wear mechanisms: gently degradation due to persistent intergranular contact with tangential displacements accumulation when the grains go upwards inside the drum, and an aggressive but short degradation due to impact after a phase of free fall.

In order to estimate the amount of wear produced in a contact, it is necessary to establish which fraction of the contact energy is absorbed by each body. Since the main goal is to obtain a global approximation of the wear, in the case of ballast-ballast contacts it is acceptable to consider that each grain absorbs half of the energy.
When the ballast is in contact with the drum, the relative displacements are high due to the lower friction coefficient, leading to a higher friction work since it is calculated, following Archard equation, over $f_{n}$ and normal forces do not change. However, this friction energy is mostly absorbed by the drum in the form of heat, leading to a lower degradation of the ballast grains than that due to contacts between grains. Following empirical observations in other MDA tests with only one grain and metal balls, the amount of energy in ballastmetal contacts causing grain degradation can be estimated between $10 \%$ and $15 \%$ of the friction energy of the contact. For this paper, $15 \%$ is considered.

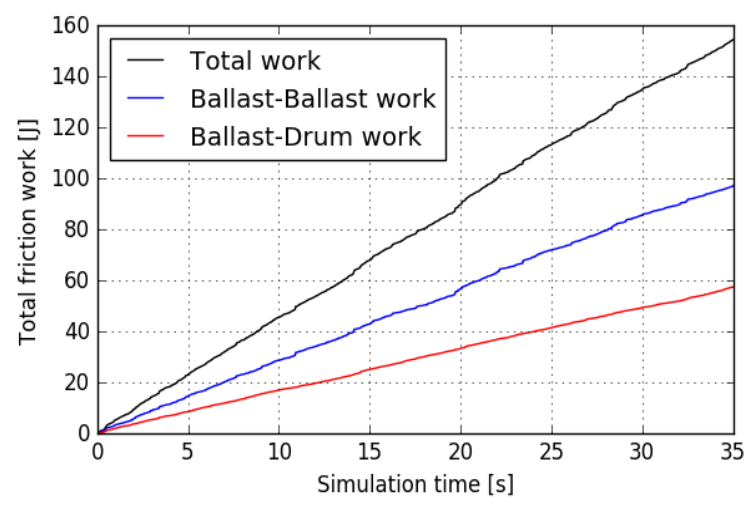

Fig. 2. Evolution of the friction work absorbed by 109 ballast grains along the NSCD simulation of a Micro-Deval test.

Figure 2 shows the amount of friction work absorbed by grains along the simulation time in each type of contact. Due to the homogeneity of the test throughout time (conditions not changing and contacts happening at the same rate), the evolution of the friction work is linear, as expected. Taking into account all types of contacts, the friction work rate is constant and equal to $4.4 \mathrm{~J} / \mathrm{s}$ absorbed by all ballast grains.

\subsection{Experimental campaign}

The data extracted from the numerical simulation needs to be compared to the actual experimental test and the amount of generated fines. This test is performed without water to be as close as possible to the simulation conditions. In order to extract the curve of mass loss during the test, the drum is stopped at intermediate stages. Grains are then cleaned and the sample is weighted. Particles bigger than $1.6 \mathrm{~mm}$ are extracted and weighted apart, so the amount of generated fines can be measured as the difference between the weight of the sample before and after the test minus the amount of small particles bigger than $1.6 \mathrm{~mm}$.

Figure 3 shows the rate of generation of fine particles during the test, stopping the drum every 5 and 20 minutes with three different ballast samples. This curve is plotted for top-quality granite used in high-speed lines and for granite for conventional lines. A curve fit is added to each set of experimental points. This function has not been chosen for any particular physical reason but as a best-fit choice, which has turned out to be a power law for all three Micro-Deval tests. 


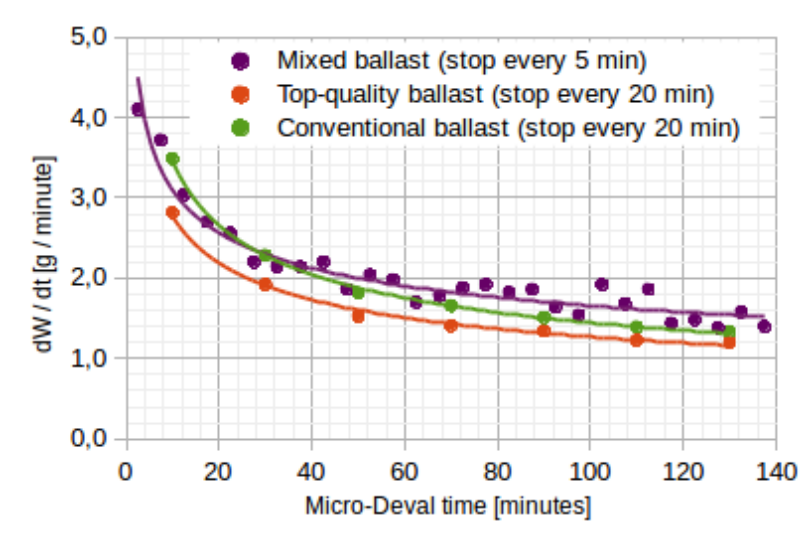

Fig. 3. Rate of fines production during a Micro-Deval test.

Two well-distinguished phases are identified: at the beginning of the test, the wear rate is higher, but it decreases rapidly following approximately the fitted power law. After the first hour of test, the production of fines tends to stabilise to a fixed wear rate of $1.2 \sim 1.3 \mathrm{~g}$ / minute (Table 1).

Table 1. Production of fines during a Micro-Deval test.

\begin{tabular}{|l|c|c|}
\hline & $\begin{array}{c}\text { Top-quality } \\
\text { ballast }\end{array}$ & $\begin{array}{c}\text { Conventional } \\
\text { ballast }\end{array}$ \\
\hline First $20 \mathrm{~min}$ & $2.82 \mathrm{~g} /$ minute & $3.49 \mathrm{~g} /$ minute \\
\hline Last $20 \mathrm{~min}$ & $1.20 \mathrm{~g} /$ minute & $1.33 \mathrm{~g} /$ minute \\
\hline
\end{tabular}

This quasi-linear production of fines observed after the initial stages of the test is coherent with the constant rate of friction work extracted from the NSCD simulations.

From a macro-scale point of view, the wear coefficient $\mathrm{k} / \mathrm{H}$ of the Archard model is not constant at the beginning of the test but it can actually be considered as constant after the first hour initial phase.

\subsection{Linking results}

Taking into account the friction work extracted from NSCD simulations and the production of fines observed in the experimental tests, it is then possible to link both results using Archard's model to get a first approximation of the wear coefficient.

After the first phase of the MDA test, an averaged constant wear rate of $1.3 \mathrm{~g} /$ minute can be assumed. Using the work rate extracted from the NSCD simulations, $k / H$ will have an approximate value of $1.8 \cdot 10^{-9} \mathrm{~Pa}^{-1}$. Wear production will be given by:

$$
W=(k / H) \cdot f_{n} \cdot s=1.8 \cdot 10^{-9} \cdot E_{f r}
$$

where $W$ is the volume of fine particles generated in $\mathrm{m}^{3}$ and $E_{f r}$ is the friction energy absorbed by the grain.

This expression, though, is only valid after the initial phase of degradation of the grains. In order to better understand the initial degradation and to be able to assess when the constant rate is reached, it is necessary to study how the morphology of the grain evolves during the process.

\section{Evolution of morphology}

Grain morphology plays a key role in the behaviour of the ballast layer as well as in the production of fine particles. Some authors [8] use three different scales to characterize shape and texture. At large scales, sphericity, elongation and flatness are usually used to provide a general idea of the grain shape. The intermediate scale is focused on describing the roundness of the grain and sharpness of angles and borders, and it is usually described using the parameter called angularity. Finally, roughness is the parameter used to describe, at the micro-scale, the surface texture.

Large scale parameters are classically calculated using the three characteristic lengths (shortest, intermediate and longest) of a rectangular prism in which the grain is inscribed $[9,10]$ : sphericity $(\psi)$, shape factor $(S F)$, flatness ratio $(F R)$, elongation ratio $(E R)$ and flatelongation ratio $(F E)$. For the intermediate parameters, angularity and form indexes are defined using harmonic analysis [11]. Finally, surface roughness is assessed by scalar parameters quantifying the height of the surface points relative to a reference line (average roughness $R_{a}$, RMS roughness $R_{q}$, total roughness $\left.R_{t}\right)$ or plane $\left(S_{a}, S_{q}\right.$, $S_{t}$ ) [12]. In addition, a fractal approach to roughness is considered $(D)$ [13].

In order to study the evolution of the different morphology parameters, five ballast grains have been identified and submitted to a more aggressive MDA test: $3 \mathrm{~h}$ of test with only 10 grains so the higher intensity of the impacts produce accelerated erosion. Before and after the MDA test, the samples have been scanned using $\mathrm{X}$-ray tomography to obtain a $3 \mathrm{D}$ image of the whole grain, and with a laser profilometer over one flat face of the grain to assess the roughness.

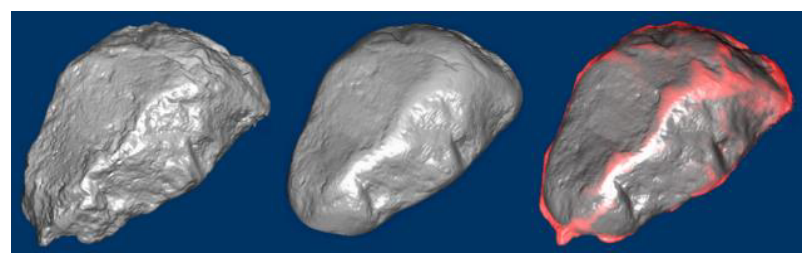

Fig. 4. X-ray images of a grain (sample S12) before and after a Micro-Deval Attrition test, and a comparison of both images highlighting the eroded areas.

In figure 4 , it can be observed how sharp corners disappear first, due to either wear or breakage of weaker parts, reducing considerably the angularity of the grain. It is also shown how roughness is dramatically reduced leaving a smooth surface. However, the general shape (elongation, sphericity, etc.) of the grain remains without remarkable changes.

Figure 5 shows the evolution in percentage of all shape parameters in comparison with their initial values. It is clearly seen how the angularity of all grains decreases in a significant amount. Large scale parameters, however, remain with approximately the same values. These parameters should tend to values close to 1 if the erosion is more aggressive, since the grains tend to reach a spherical-ellipsoidal shape. However, the MDA test is not able to reproduce such a 
big amount of erosion and ballast in railway tracks is actually substituted much before these erosion levels are reached.

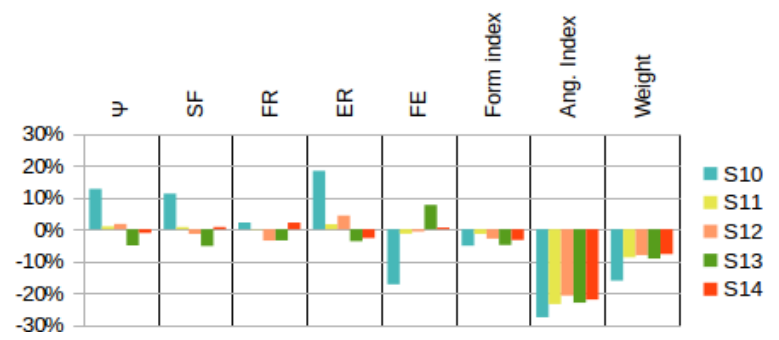

Fig. 5. Evolution of the different shape parameters. S1X is the name of the different grain samples.

Figure 6 shows the evolution in percentage of the different roughness parameters and fractal dimension. As expected, these parameters are clearly reduced during the process (except for sample S13, which presented an already relatively smooth surface before the erosion). Empirical observations show evidence that roughness is lost during the first minutes of the MDA test, corresponding to the higher production of fine particles at the beginning of the test.

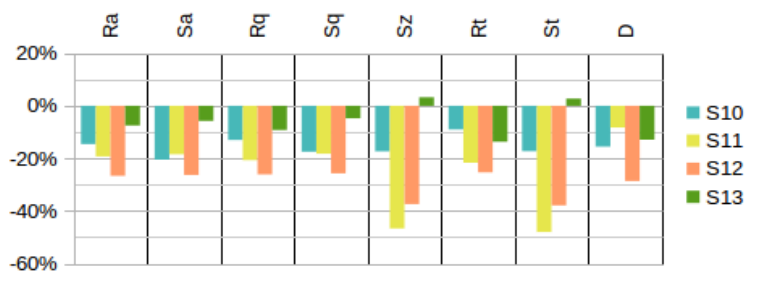

Fig. 6. Evolution of the different roughness parameters.

Some studies [14] confirm the existence of at least two phases in the wear process: first angularity is progressively lost without changing the main axis dimensions and it is afterwards that the general shape is slowly rounded. The results shown in this paper suggest the existence, for sharp and rough grains, of an initial phase corresponding to a fast degradation at early stages, which leads to a three-phase wear process corresponding to the three morphology scales.

Friction wear of ballast grains is a local phenomenon concerning only the first two phases: the micro-texture of the grain and its angularity. This behaviour is in accordance with the two phases observed in the rate of generation of fine particles during the MDA test.

\section{Conclusions}

Discrete Element simulations (NSCD) of the MicroDeval Attrition test have shown that the amount of energy absorbed by the grains follow a linear tendency over time. However, experimental tests have demonstrated that the production of fine particles is not linear during the whole test, but it is more aggressive at the beginning and becomes almost linear after the first half of the test producing about $1.3 \mathrm{~g} /$ minute.

Following Archard, it is possible to relate the linear phase of the wear process to the wear coefficient $k$. In order to understand this wear process, grain morphology has been tracked and analysed.

The morphology study has shown that the smaller the scale is the faster the parameters are smoothed. Hence large scale parameters have a very slow evolution, depending to a large extent on the grains crushing. The intermediate scale, mainly represented by angularity, plays a very important role in the evolution of shape since sharp angles and edges are rounded and weak pointed tips break easily. Finally, micro roughness is lost during the very first minutes of the test.

This evolution is coherent with the experimental curve of mass loss. The higher production of fine particles at the beginning of the Micro-Deval Attrition test can be related to the polishing of the surface texture and weaker edges and vertices, losing very quickly the roughness and reducing faster the angularity. Afterwards, the process becomes more linear since faces are already polished and edges and vertices present larger surfaces to resist the contact forces. From this point, angularity is reduced in a slower but constant process leading to an almost linear production of fines.

As a perspective for further studies, the morphology description of a grain, especially concerning roughness and angularity, should be associated to a specific point in the wear rate curve.

The authors gratefully acknowledge Denis Tunkin for his contribution to the study of shape evolution during his Master thesis. The Laboratoire 3SR is part of the LabEx Tec 21 (Investissements d'Avenir - grant agreement $\mathrm{n}^{\circ}$ ANR-11LABX-0030).

\section{References}

1. AFNOR, NF EN 1097-1 (Saint Denis, 1996)

2. AFNOR, NF EN 13450 (Saint Denis, 2003)

3. J.F. Archard, J. Appl. Phys., 24, 981-988 (1953)

4. I. Deiros, C. Voivret, G. Combe, F. Emeriault, Proc. of the Third International Conference on Railway Technology (Civil-Comp Press, UK, 2016)

5. I. Deiros, C. Voivret, G. Combe, F. Emeriault, Procedia Engineering, 143, 1016-1023 (2016)

6. M. Jean, Computer Methods in Applied Mechanics and Engineering, 177(3-4), 235-257 (1999)

7. J. Hans, M. Boulon, Int. J. Numer. Anal. Meth. Geomech., 27(6), 513-548 (2003)

8. J.K. Mitchell, K. Soga, Fundamentals in Soil Behavior (John Wiley \& Sons, New Jersey, 2005)

9. W.C. Krumbein, J. Sedimentary Petrology, 11, 64$72(1941)$

10. R.D. Barksdale, M.A. Kemp, W.J. Sheffield, J.L. Hubbard, Transp. Research Record, 1301, 107-116 (1991)

11. E.J. Garboczi, Cement and Concrete Research, 32(10), 1621-1638 (2002)

12. B. Bhushan, Modern Tribology Handbook (CRC Press, USA, 2000)

13. A. Majumdar, B. Bhushan, J. Tribology, 112(2), 205-216 (1990)

14. G. Domokos, D.J. Jerolmack, A.A. Sipos, Á. Török, PLOS one, 9(2), e88657 (2014) 